\title{
Urinary Soluble Chemokine (C-X-C Motif) Ligand 16 (CXCL16) and Urinary Neutrophil Gelatinase Associated Lipocalin (NGAL) as Biomarkers of Activity in Children and Adolescents with Lupus Nephritis
}

\author{
Mohamed A El-Gamasy ${ }^{1}$, Maher Abdelhafez and Hend Abdelnabi
}

Department of Pediatrics, Faculty of Medicine, Tanta University, Egypt

\begin{abstract}
Objectives: One of the challenges of treating patients with lupus nephritis (LN) is to assess disease activity and predict its outcome. Since renal biopsy cannot be performed routinely, early biomarkers are needed. The aim of this study was to measure urinary Neutrophil Gelatinase Associated Lipocalin (NGAL) and urinary soluble chemokine (C-X-C motif) Ligand 16 (CXCL16) levels in children and adolescents with Systemic lupus erythematosus (SLE) and investigate whether they are elevated in active LN.

Methods: The study was conducted on 80 patients diagnosed as SLE by Systemic Lupus International Collaborating Clinics (SLICC) criteria and 60 apparently healthy subjects as controls. Global and renal disease activity was evaluated by Systemic Lupus Erythematosus Disease Activity Index (SLEDAI) and r SLEDAI respectively. Urinary NGAL and Urinary CXCL16 were measured for all subjects by ELISA. Renal biopsy was done for all cases at initial diagnosis and was graded using ISN/RPS classification.

Results: Urinary NGAL and CXCL16 were higher in patients than in the controls. Their levels were higher in patients with LN than those without LN. Urinary NGAL had higher sensitivity and specificity than urinary CXCL16 as early predictor of LN. There were significant positive correlations between urinary NGAL levels and 24 hour urinary proteins and SLEDAI and there were also significant positive correlations between urinary CXCL16 levels and 24 hour urinary proteins and SLEDAI.
\end{abstract}

Conclusions: UNGAL and CXCL16 were reliable indicators of the activity of $L N$ pointing to the underlying renal pathology.

\section{Keywords: SLE; Urinary NGAL; CXCL16; Nephritis}

\section{Introduction}

Systemic lupus erythematosus (SLE) is a disease of unknown aetiology in which tissues are damaged by autoantibodies and immune complexes. Lupus nephritis (LN) is considered the most frequent manifestation of SLE and can be present in 60\% of SLE patients [1].

Kidney biopsy remains the mainstay for diagnosis of LN and is usually presented by an abnormal urinary findings e.g., sediment, proteinuria or elevated serum creatinine. These markers do not correlate with histopathological diagnosis. Kidney damage is known to precede the appearance of proteinuria, elevation of serum creatinine or abnormal urine sediments leading to a delay in management of $\mathrm{LN}$, its flares and assessment of response for therapy, thus leading to more morbidity and mortality [2].

Disease biomarker is defined as cellular, biochemical, molecular or genetic alteration by which a normal or abnormal biologic process can be recognized and/or monitored and that may have diagnostic or prognostic utility. Reliable biomarkers may help evaluation of disease activity, identify patients at risk for organ damage or recurrent flares, and facilitate early and accurate evaluation of responses to treatment. A parameter to serve as a biomarker should be biologically plausible and relevant to disease pathogenesis, reflect changes in disease activity, and be simple to measure for routine use [2].

Neutrophil Gelatinase Associated Lipocalin (NGAL) also known as Lipocalin-2 is a $25-\mathrm{kDa}$ acute phase protein originally purified from human neutrophils, and later found to be induced in most tissues exposed to micro-organisms and in epithelial cells during inflammation [3]. NGAL was identified by microarray analysis as one of the earliest and most robustly induced genes and proteins in the kidney after ischemic or nephrotoxic injury in both animals and humans and was easily detected in the urine and blood soon after acute kidney injury [3]. Urinary NGAL was proven as excellent biomarker of concurrent lupus nephritis activity [4].

Serum soluble chemokine (C-X-C motif) Ligand 16 (CXCL16) is expressed on macrophages and dendritic cells (DCs) and plays important roles including T-cell and NK-cell recruitment and intercellular adhesion mediated by interactions with its receptor, CXCL16 receptor is expressed on the surface of DCs, macrophages and smooth muscle cells [4]. CXCL16 is expressed by a variety of tissues e.g., inflamed vasculature, bronchial epithelium, rheumatoid joints, liver, and kidney. In immune systems, it can attract $\mathrm{T}$ cells and supports a prolonged interaction between antigen-presenting cells (APCs) and activated T cells. CXCL16 may be involved in the pathogenesis of rheumatoid arthritis, Behcet's disease and gouty arthritis [4].

*Corresponding author: Dr. Mohamed Abdelaziz El-Gamasy, Department of Pediatrics, Faculty of Medicine, Tanta University Hospital, Tanta University, El Giesh Street, Tanta, Gharbia, Egypt, Tel: +201208136076; E-mail: mgamsy@gmail.com

Received September 05, 2017; Accepted September 14, 2017; Published September 20, 2017

Citation: El-Gamasy MA, Abdelhafez M, Abdelnabi H (2017) Urinary Soluble Chemokine (C-X-C Motif) Ligand 16 (CXCL16) and Urinary Neutrophil Gelatinase Associated Lipocalin (NGAL) as Biomarkers of Activity in Children and Adolescents with Lupus Nephritis. Clin Med Biochem 3: 135. doi:10.4172/24712663.1000135

Copyright: ( 2017 El-Gamasy MA, et al. This is an open-access article distributed under the terms of the Creative Commons Attribution License, which permits unrestricted use, distribution, and reproduction in any medium, provided the original author and source are credited. 
Citation: El-Gamasy MA, Abdelhafez M, Abdelnabi H (2017) Urinary Soluble Chemokine (C-X-C Motif) Ligand 16 (CXCL16) and Urinary Neutrophil Gelatinase Associated Lipocalin (NGAL) as Biomarkers of Activity in Children and Adolescents with Lupus Nephritis. Clin Med Biochem 3: 135. doi:10.4172/2471-2663.1000135

Page 2 of 6

CXCL16 was recently reported to be selectively up-regulated in a wide range of tissues in response to damage, especially in kidney [4]. CXCL16 has been documented to be increased within the kidneys, sera and urine of SLE patients and seem to correlate with disease activity, although their clinical utility in predicting disease activity in LN remains unclear [5].

We aimed in this research to measure urinary NGAL and CXCL16 levels in children and adolescents with SLE and investigate whether they were elevated in active SLE patients and to verify their role in early diagnosis of lupus nephritis.

\section{Methods}

\section{Design of the study and setting}

The prospective, case-control and cross-sectional study was conducted after approval from the ethical committee of the Faculty of Medicine, Tanta University and informed written parental consents on 80 patients diagnosed as SLE (including both already known previously diagnosed cases who were on treatment and newly diagnosed cases who were not starting treatment). They were selected from the inpatient department and the out-patient clinic of the Pediatric Nephrology Unit of the Pediatric Department, Tanta University Hospital between July 2016 and July 2017.

\section{Inclusion criteria}

All patients previously (already diagnosed before and were on treatment) or currently (recently diagnosed) fulfilled the 2012 revised Systemic Lupus International Collaborating Clinics (SLICC) criteria for the diagnosis of SLE [6].

They were 70 females $(87.50 \%)$ and 10 males. F: $M$ ratio (7:1). Their ages ranged between 4 and 18 years, with a mean of $12.07 \pm 3.35$ years. 60 apparently healthy subjects were carefully chosen, matched for age and sex as controls. They were 52 females $(86.67 \%)$ and 8 males, F: M ratio $(6.5: 1)$. Their age ranged between 5 and 17 years, with a mean of $12.32 \pm 3.23$ years, acted as a healthy control group.

\section{All subjects were subjected to}

\section{Full history taking:}

Thorough clinical examination: Global disease activity was evaluated according to the Systemic Lupus Erythematosus Disease Activity Index (SLEDAI) score which is a validated disease activity measure for childhood-onset SLE with a total score of $0-105$. The tool consists of 24 weighted items grouped into the following nine domains: central nervous system (CNS), vascular, renal, musculoskeletal, serosal, dermal, immunological, constitutional and hematological diseases. Renal disease activity was evaluated according to the renal Systemic Lupus Erythematosus Disease Activity Index (rSLEDAI) [7].

\section{Laboratory investigations}

Routine laboratory investigations: Complete blood picture (CBC) Erythrocyte sedimentation rate (ESR), serum creatinine, 24 hour urine proteins, serum C3, C4, ANA and Anti-dsDNA.

Urinary NGAL and urinary CXCL16: They were measured by the enzyme-linked immunosorbent assay.

\section{Sampling}

All urine samples were diluted 1:1 for the ELISA and the concentrations of the respective molecules were ascertained from standard curves constructed using manufacturer-supplied standards.
In the Biovendor Human NGAL ELISA, Standards, Quality Controls and samples were incubated in micro plate wells with polyclonal antihuman NGAL antibody. After one hour incubation and washing, biotin-labelled polyclonal anti-human NGAL antibody was added and incubated with captured NGAL for one hour. After another washing, streptavidin-HRP conjugate was added. After 30 minutes incubation and the last washing step, the remaining conjugate was allowed to react with the substrate solution (TMB). The reaction was stopped by addition of acidic solution and absorbance of the resulting yellow product was measured spectrophotometrically at $450 \mathrm{~nm}$. Urinary CXCL16 concentrations were quantified by using a double-ligand ELISA methodology, according to the manufacturer's instructions (chemokine domain, R\&D Sys-tem, Minneapolis, MN, USA).

\section{Renal biopsy}

The kidney biopsies were done for all cases at initial diagnosis to determine the severity of Lupus Nephritis (LN). The pathologic changes present on renal biopsy help guiding treatment decisions and may be predictive of long-term kidney survival; the histological findings are graded using the International Society of Nephrology/ Renal Pathology Society (ISN/RPS) Classification. Class I and II used for purely mesangial involvement (I, mesangial immune deposits without mesangial hypercellularity; II, mesangial immune deposits with mesangial hypercellularity), class III for focal glomerulonephritis (involving $<50 \%$ of total number of glomeruli) with subdivisions for active and sclerotic lesions; class IV for diffuse glomerulonephritis (involving $>$ or $=50 \%$ of total number of glomeruli) either with segmental (class IV-S) or global (class IV-G) involvement, and also with subdivisions for active and sclerotic lesions; class $\mathrm{V}$ for membranous lupus nephritis; and class VI for advanced sclerosing lesions [8].

\section{Statistical analysis}

Patient's data were tabulated and processed using SPSS V16 software statistical computer package. The difference between parametric data of groups analyzed via Student's t-test, ANOVA test. Chi-square test (X2) was used for comparison of frequencies. Spearman's correlation coefficient ( $r$ ) was used to assess the degree of association between 2 continuous variables. To assess the diagnostic value we calculated the following indices: cut off value, accuracy, sensitivity, specificity, positive predictive value (PPV) that is the proportions of the positive results that are true positive and negative predictive value (NPV) that is the proportions of the negative results that are true negative. A value of $(\mathrm{P}<0.05)$ considered significant [9].

\section{Results}

$28(35 \%)$ of the selected patients were recently diagnosed cases and $52(65 \%)$ were already known and treated cases. The disease duration in the latter ranged from 12-120 months with a mean \pm SD of $42.4 \pm 38.2$.

The demographical and laboratory data of the studied groups were summarized in Table 1, the total number of the SLE patients were 80, 70 females $(87.50 \%)$ and 10 males. F: $\mathrm{M}$ ratio (7:1). Their ages ranged from 4 to 18 years, with a mean of $12.07 \pm 3.35$ years. 60 apparently healthy subjects were carefully chosen, matched for age and sex with the SLE patients. They were 52 females (86.67\%) and 8 males, F: M ratio (6.5:1). Their age ranged from 5 to 17 years, with a mean of $12.32 \pm 3.23$ years, with no significant differences between the patients and controls.

SLEDAI score has been used in our study as a predictor of disease activity. The mean global SLEDAI score was $28.6 \pm 21.460$, renal SLEDAI was $9.68 \pm 4.57$ and extra-renal SLEDAI was $17.89 \pm 9.34$. 
Citation: El-Gamasy MA, Abdelhafez M, Abdelnabi H (2017) Urinary Soluble Chemokine (C-X-C Motif) Ligand 16 (CXCL16) and Urinary Neutrophil Gelatinase Associated Lipocalin (NGAL) as Biomarkers of Activity in Children and Adolescents with Lupus Nephritis. Clin Med Biochem 3: 135. doi:10.4172/2471-2663.1000135

The mean concentrations of urinary NGAL $(8.9 \pm 3.56 \mathrm{ng} / \mathrm{dl})$ and urinary CXCL16 $(10.67 \pm 3.67 \mu \mathrm{g} / \mathrm{L})$ in SLE patients were significantly higher than the controls $(\mathrm{P}<0.05)$.

There was no statistically significant sex differences in urinary NGAL and CXCL16 in the studied patients group $(\mathrm{P}>0.05)$. Renal biopsies at initial diagnosis evaluated according ISN lupus nephritis grading system as shown in Table 2. ISN Classes I (50\%) and III (35\%) were the commonest findings.

Table 3 compared also between the mean levels of urinary NGAL and urinary CXCL16 in SLE patients with renal affection according to renal biopsy results, there was significant difference between mean levels of urinary NGAL and urinary CXCL16 in SLE patients with renal affection of different classes according to renal biopsy results $(\mathrm{p}<0.05)$.

Table 4 and Figure 1 summarized validity of urinary NGAL and urinary CXCL16 in early prediction of lupus nephritis, urinary NGAL had higher sensitivity and specificity than urinary CXCL16 in early prediction of lupus nephritis.

At a cut off value $\geq 8.28$ (ng/dl), Urinary NGAL had sensitivity of $95 \%$ with specificity $90 \%$, PPV 1.06, NPV 0.98 and accuracy (AUC) 0.94 while at a cut off value $\geq 8.13(\mu \mathrm{g} / \mathrm{L})$, Urinary CXCL16 had sensitivity of $85 \%$ with specificity $80 \%$, PPV 0.71 , NPV 1.56 and accuracy (AUC) 0.91 .

Analysis of the correlations between NGAL, CXCL16 and other variables including age, $\mathrm{Hb} \%$, TLC, Platelet count, ESR, serum

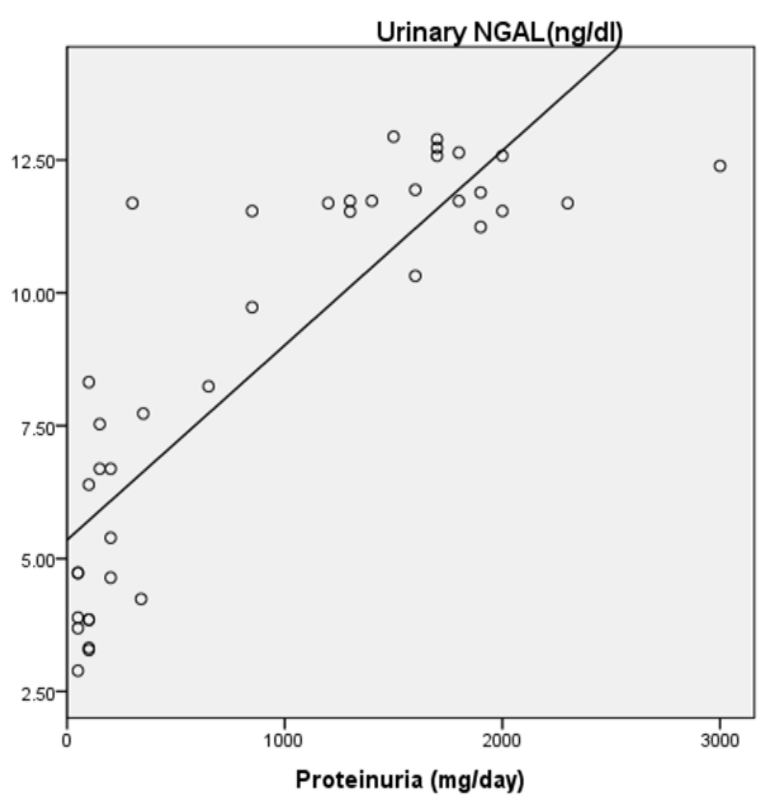

Figure 1: Correlation between urinary NGAL and urinary protein in studied patients.

\begin{tabular}{|c|c|c|c|}
\hline Parameters & Patients & Controls & $P$ value \\
\hline Number of patients & 80 & 60 & - \\
\hline Female/male ratio & $7 / 1$ & $6.5 / 1$ & - \\
\hline Females: number (percent) & $70(87.5 \%)$ & $52(86.67 \%)$ & 0.93 \\
\hline Age (years): mean \pm SD & $12.07 \pm 3.35$ & $12.32 \pm 3.23$ & 0.86 \\
\hline $\begin{array}{c}\text { Global SLEDAI } \\
\text { Renal SLEDAI } \\
\text { Extra-renal SLEDAI }\end{array}$ & $\begin{array}{c}28.6 \pm 21.46 \\
9.68 \pm 4.57 \\
17.89 \pm 9.34\end{array}$ & $\begin{array}{l}- \\
- \\
-\end{array}$ & $\begin{array}{l}- \\
- \\
-\end{array}$ \\
\hline Serum creatinine $(\mathrm{mg} / \mathrm{dl})$ mean $\pm \mathrm{SD}$ & $0.98 \pm 0.55$ & $0.61 \pm 0.2$ & 0.001 \\
\hline $\mathrm{Hb}(\mathrm{g} / \mathrm{dl})$ mean $\pm \mathrm{SD}$ & $9.11 \pm 1.65$ & $11.9 \pm 1.46$ & 0.001 \\
\hline TLC $\left(\times 10^{3} / \mathrm{mm}^{3}\right)$ mean $\pm \mathrm{SD}$ & $2.86 \pm 1.14$ & $8.51 \pm 2.73$ & 0.001 \\
\hline Platelets $\left(\times 10^{3} / \mathrm{mm}^{3}\right)$ mean $\pm \mathrm{SD}$ & $186.25 \pm 97.92$ & $255.5 \pm 101.186$ & 0.034 \\
\hline ESR $1^{\text {st }}$ hour $(\mathrm{mm})$ mean \pm SD & $74.1 \pm 31.13$ & $13.6 \pm 8.10$ & 0.001 \\
\hline ESR $2^{\text {nd }}$ hour $(\mathrm{mm})$ mean $\pm \mathrm{SD}$ & $105.25 \pm 32.90$ & $18.25 \pm 14.30$ & 0.001 \\
\hline $24 \mathrm{~h}$ urinary proteins (mg/day) mean $\pm \mathrm{SD}$ & $981 \pm 862.25$ & $45.78 \pm 24.22$ & 0.001 \\
\hline Serum C3 (mg/dl) mean \pm SD & $52.19 \pm 25.79$ & $135.8 \pm 9.568$ & 0.001 \\
\hline $\begin{array}{c}\text { Serum C4 }(\mathrm{mg} / \mathrm{dl}) \text { mean } \pm \text { SD } \\
\text { ANA }(\mathrm{u} / \mathrm{ml}) \text { mean } \pm \text { SD } \\
\text { Anti-dsDNA }(\mathrm{u} / \mathrm{ml}) \text { mean } \pm \text { SD } \\
\text { Urinary NGAL }(\mathrm{ng} / \mathrm{dl}) \text { mean } \pm \text { SD } \\
\text { Urinary CXCL16 }(\boldsymbol{\mu g} / \mathrm{L}) \text { mean } \pm \text { SD }\end{array}$ & $\begin{array}{c}8.09 \pm 6.98 \\
101.23 \pm 83.27 \\
227.93 \pm 165.46 \\
8.9 \pm 3.56 \\
10.67 \pm 3.67\end{array}$ & $\begin{array}{c}25.15 \pm 3.1 \\
12.46 \pm 20.33 \\
10.83 \pm 4.35 \\
2.26 \pm 1.95 \\
4.71 \pm 1.06\end{array}$ & $\begin{array}{l}0.001 \\
0.001 \\
0.001 \\
0.001 \\
0.001\end{array}$ \\
\hline
\end{tabular}

Student(s) test used for comparing mean \pm SD of patients and control groups; Chi square $(\times 2)$ test used for comparing no (\%) of patients and control groups; NGAL: Neutrophil gelatinase associated Lipocalin; CXCL16: CXC chemokine ligand 16

Table 1: Demographic and laboratory data of the studied patients and controls.

\begin{tabular}{|c|c|c|c|}
\hline Renal biopsy classes & $\begin{array}{c}\text { Urinary NGAL (ng/d) mean } \\
\pm \text { SD }\end{array}$ & $\begin{array}{c}\text { Urinary CXCL16 ( } \mathbf{\mu g} / \mathbf{L}) \text { mean } \\
\pm \text { SD }\end{array}$ & N \\
\hline Lupus nephritis class I & $5.86 \pm 2.84$ & $9.09 \pm 3.11$ & 40 \\
\hline Lupus nephritis class II & 9.96 & 9.85 & 2 \\
\hline Lupus nephritis class III & $11.36 \pm 1.19$ & $10.79 \pm 3.25$ & 28 \\
\hline Lupus nephritis class IV & $12.14 \pm 0.64$ & $15.19 \pm 2.21$ & 10 \\
\hline Lupus nephritis class V & 0 & 0 & 0 \\
\hline P value & 0.001 & 0.004 & - \\
\hline
\end{tabular}

NGAL: Neutrophil gelatinase associated lipocalin; CXCL16: CXC chemokine ligand 16; ANOVA test used for comparing mean \pm SD of different classes of patients; NB. On comparing between each subgroup pairs (class I, class II, class III, and class VI, there was no statistical significant difference between each pairs regarding NGAL and CXCL16 $(P>0.05)$

Table 2: Comparison between levels of urinary NGAL and urinary CXCL16 in SLE patients with renal affection according to renal biopsy results. 
Citation: El-Gamasy MA, Abdelhafez M, Abdelnabi H (2017) Urinary Soluble Chemokine (C-X-C Motif) Ligand 16 (CXCL16) and Urinary Neutrophil Gelatinase Associated Lipocalin (NGAL) as Biomarkers of Activity in Children and Adolescents with Lupus Nephritis. Clin Med Biochem 3: 135. doi:10.4172/2471-2663.1000135

\begin{tabular}{|c|c|c|}
\hline & Urinary NGAL $(\mathbf{n g} / \mathbf{d l})$ & Urinary CXCL16 $(\boldsymbol{\mu g} / \mathbf{L})$ \\
\hline Cut off value & $8.28 \mathrm{ng} / \mathrm{dl}$ & $8.13 \mu \mathrm{\mu g} / \mathbf{L}$ \\
\hline Sensitivity & $95 \%$ & $85 \%$ \\
\hline Specificity & $90 \%$ & $80 \%$ \\
\hline Accuracy(AUC) & 0.94 & 0.91 \\
\hline PPV & 1.06 & 0.71 \\
\hline NPV & 0.98 & 1.56 \\
\hline
\end{tabular}

NGAL: Neutrophil gelatinase associated Lipocalin; CXCL16: CXC chemokine ligand 16; AUC: area under the curve; PPV: positive predictive value; NPP: Negative predictive value

Table 3: Validity of urinary NGAL and urinary CXCL16 in early prediction of lupus nephritis.

\begin{tabular}{|c|c|c|c|c|}
\hline & 24 hours collected urine $(\mathbf{m g})$ & \multicolumn{2}{c|}{ SLEDAI } \\
\cline { 2 - 5 } & $\mathbf{r}$ & P-value & $\mathbf{r}$ & P-value \\
\hline Urinary NGAL $(\mathrm{ng} / \mathrm{dl})$ & 0.732 & 0.001 & 0.359 & 0.001 \\
\hline Urinary CXCL16 $(\mu \mathrm{g} / \mathrm{L})$ & 0.470 & 0.001 & 0.17 & 0.35 \\
\hline
\end{tabular}

NGAL: Neutrophil gelatinase associated Lipocalin; NGAL: Neutrophil Gelatinase Associated Lipocalin; CXCL16: CXC Chemokine Ligand 16

Table 4: Correlations between urinary NGAL, urinary CXCL16, urinary protein/24 hours collected urine and SLEDAI.

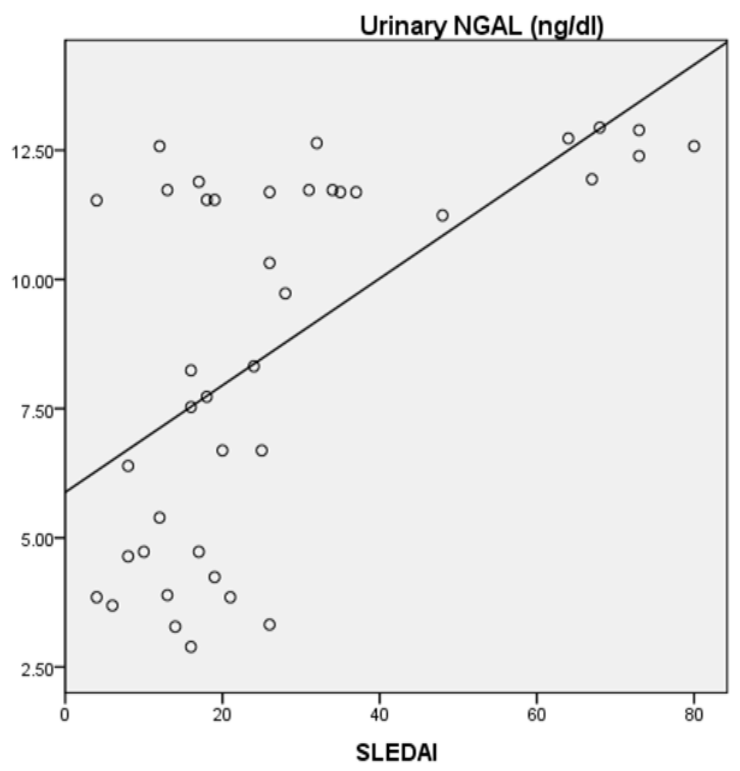

Figure 2: Correlation between urinary NGAL and SLEDAI in the studied patients.

creatinine, 24 hour urine proteins, serum C3, C4, ANA and AntidsDNA were done and there were no statistically significant correlation between NGAL/CXCL16 and patients' age, Hb \%, TLC, Platelet count, ESR, serum creatinine, 24 hour urine proteins, serum C3, C4, ANA and Anti-dsDNA $(\mathrm{P}>0.05)$ in patient group.

Regarding correlations between urinary NGAL, urinary CXCL16, urinary protein/ 24 hours collected urine and SLEDAI. There was a significant positive correlation between urinary NGAL levels and 24 hour urinary proteins $(\mathrm{r}=0.732, \mathrm{P}=0.001)$ and between urinary NGAL levels and SLEDAI $(r=0.359, \mathrm{P}=0.001)$ (Figure 2) and there was a significant positive correlation between urinary CXCL16 levels and 24 hour urinary proteins $(\mathrm{r}=0.470, \mathrm{P}=0.001)$ and between urinary $\mathrm{CXCL} 16$ levels and SLEDAI ( $\mathrm{r}=0.739, \mathrm{P}=0.001)$ (Figure 3 ).

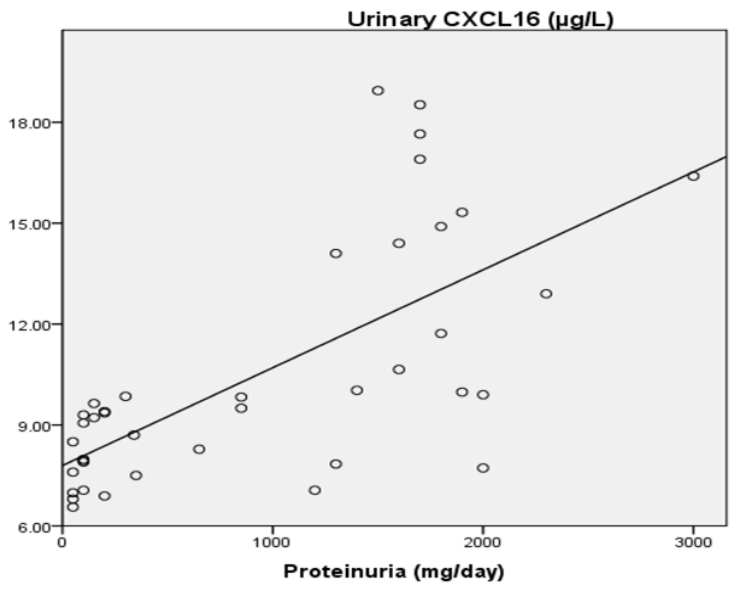

Figure 3: Correlation between urinary CXCL16 and urinary protein in studied patients.

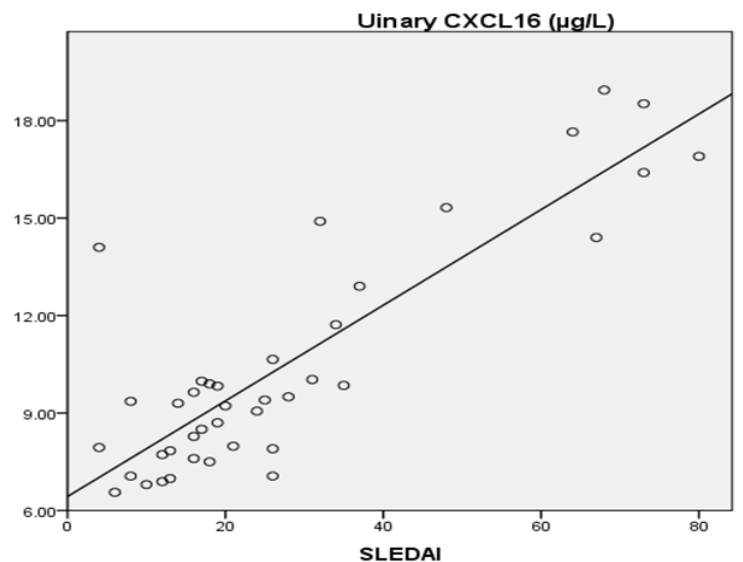

Figure 4: Correlation between urinary CXCL16 and SLEDAI in studied patients.

\section{ROC Curve}

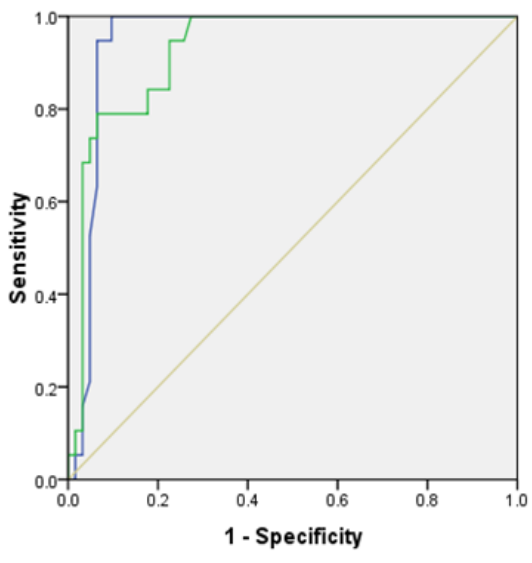

Source of the Curve - NGAL RXelerence Lin

Diagonal segments are produced by ties.

Figure 5: ROC curves of urinary NGAL and CXCL16 for prediction of activity of lupus nephritis. 
Citation: El-Gamasy MA, Abdelhafez M, Abdelnabi H (2017) Urinary Soluble Chemokine (C-X-C Motif) Ligand 16 (CXCL16) and Urinary Neutrophil Gelatinase Associated Lipocalin (NGAL) as Biomarkers of Activity in Children and Adolescents with Lupus Nephritis. Clin Med Biochem 3: 135. doi:10.4172/2471-2663.1000135

Page 5 of 6

\section{Discussion}

Conventional markers of SLE disease activity fail to adequately identify active LN early [10]. Singh et al. reported that urinary vascular cell adhesion molecule-1 (VCAM-1) as acute phase reactants was a reliable indicator of the activity: chronicity ratios that mark the underlying renal pathology in lupus nephritis [1].

NGAL has been identified to be an important marker for acute kidney injury [11], tubular injuries in kidney transplant patients [11], contrast induced nephrotoxicity and cisplatin nephropathy [12], eGFR in chronic kidney disease [13]. It correlated well with residual renal function and serum creatinine in patients with autosomal dominant polycystic kidney disease [14]. The UNGAL was reported as a predictor of severity of thrombotic microangiopathies secondary to associated tubular injury [14].

Ding et al. reported that UNGAL represented an early biomarker for the degree of chronic tubulointerstitial injury in Burger disease with strong correlations between the uNGAL/u creatine ratio and both clinical and histopathological disease activity [15].

Yanaba reported that CXCL16 levels were increased in patients with systemic sclerosis (SSc) and correlated with the extent of skin sclerosis, suggesting that CXCL16 may have a role in the development of skin fibrosis in systemic sclerosis and added that blockade of CXCL16 interaction might be a potential therapeutic target in patients with SSc [16].

In the present study we aimed to measure uNGAL and CXCL16 levels in children and adolescents with SLE and to investigate whether they are elevated in active SLE patients and to verify their role in early diagnosis of lupus nephritis.

As regard to sociodemographic data of patients, girls to boys ratio was 7:1 in which it refers to significance of endocrinal factors in the clinical presentation of SLE. On comparison to other Arab researches in pediatric age, this ratio was-lower than study of Bakr [17], (12:1) and higher than study of Salah et al. [18], (2. 7:1) and Ali et al. [19], which was on Indian kids $(4: 1)$, Uziel et al. [20], which on Israeli children, (5:1), and Dunget et al. [21], in Vietnam (4:1). Difference in gender distribution might be different numbers of patients, due to differences in ethnicity or genetic factors.

Generally, children and adolescents have higher values of SLEDAI scores than adults [22]. Global and renal SLEDAI score have been used in our study as predictors of disease activity [7]. Mean global SLEDAI scores was $28.6 \pm 21.46$ and which was similar to that of Dunget et al. [21] 2012 (23.8 \pm 11.6$)$ (Figure 4).

In the current study, there was statistically significant increase in ANA and anti-dsDNA and statistically significant decrease in C3 and $\mathrm{C} 4$ in the patients as compared to healthy children these results were in agreement with Bader et al. [23].

In this study urinary levels of NGAL and serum levels of CXCL16 were analyzed in patients with concurrent renal biopsies which were performed at different stages during their follow up (Figure 5).

Lupus nephritis class I was the commonest finding on kidney biopsy in our study, 40 (50\%) of patients, $35 \%$ was with class III, $12.5 \%$ with class IV, $2.5 \%$ with class II and no patients with class V. These results were not in accordance with Pluchinotta et al. [24], or Ramirez et al. [25], who reported that class IV was the most dominant result in their histopathologies. The difference in results might be attributed to different indications of kidney biopsies in different research works.
Pluchinotta et al. [24], and Ramirez et al. [25], did biopsies only for suspected severe LN but in our present study, kidney biopsies were done routinely for all newly diagnosed SLE patients.

A cohort of Suzuki et al. was the first to show an association between NGAL and diffuse proliferative glomerulonephritis in paediatric SLE patients [26]. uNGAL and sCXCL16 were significantly higher in patients with SLE than in the control groups $(\mathrm{P}<0.05)$. Their levels were higher in SLE patients with renal affection than those without renal affection according to renal biopsy results $(\mathrm{P}<0.05)$. These results are consistent with theory that higher levels of uNGAL were found in patients with progressive CKD [27].

Our results were in agreement with Susianti et al. who concluded that uNGAL or combined u-NGAL and urinary transforming growth factor beta 1 (uTGF- $\beta 1$ ) or urinary monocyte chemoattractant protein-1 (uMCP-1) had the best sensitivity and specificity for active LN [28].

In our work, urinary NGAL showed a higher diagnostic sensitivity (95\%) and specificity (90\%) than serum CXCL16 which had a sensitivity of $85 \%$ and specificity of $80 \%$ in prediction of activity of LN.

Our results were in agreement with Qin et al. who reported that sCXCL16 levels were elevated in LN and recommended it to be used as serological marker of disease activity and added it might be used for evaluation of treatment strategies [5]

Singh et al. reported that CXCL16 was less promising marker for LN when compared to MCP-1 or VCAM-1 [1]. On comparing the values of UNGAL and sCXCL16 with corresponding renal histopathological classes in this study, both markers were increased in class IV more than class III more than class II more than class I so they tended to correlate with severity of pathological findings. This was a very promising finding.

In this work, uNGAL and sCXCL showed statistically significant positive correlations with 24-hour estimated proteinuria and SLEDAI scores. So, these two markers could refer to disease activity, monitoring and thus adequate therapy.

These correlations were in agreement with Brunner et al. who found a positive correlation between UNGAL and both disease activity and their renal histopathological findings [4].

Our results were in agreement with Torres-Salido et al. who evaluated fractional excretion (FE) of uNGAL relative to $\mathrm{FE}$ of urinary proteins ratio (FE NGAL/FE protein ratio) as it provided additional benefit to the traditional biomarkers and UNGAL to predict a proteinuric flare before worsening of rSLEDAIs and to stratify outcomes of patients with LN [29].

In study of Singh et al., they reported that uCXCL16 showed a prognostic value it had a negative correlation with chronicity index [1].

Our results differed from results of Susianti et al. who reported that active LN showed significantly reduced FE NGAL/FE protein ratios, with high specificity and sensitivity to distinguish renal from non-renal disease [28].

We concluded that urinary NGAL and CXCL16 were higher in SLE than in the control groups $(\mathrm{P}<0.05)$. Their levels were higher in SLE patients with renal affection $(\mathrm{P}<0.05)$ than those without renal affection according to renal biopsy results. So uNGAL and CXCL16 are considered as reliable indicators of the activity of $\mathrm{LN}$ which refers to the underlying histopathology of the kidney. Urinary NGAL showed a higher diagnostic sensitivity (0.95) and specificity (0.90) than urinary CXCL16. 
Citation: El-Gamasy MA, Abdelhafez M, Abdelnabi H (2017) Urinary Soluble Chemokine (C-X-C Motif) Ligand 16 (CXCL16) and Urinary Neutrophil Gelatinase Associated Lipocalin (NGAL) as Biomarkers of Activity in Children and Adolescents with Lupus Nephritis. Clin Med Biochem 3: 135. doi:10.4172/2471-2663.1000135

Page 6 of 6

It is recommended for serial monitoring of pediatric patients with LN for non-invasive markers including urine levels of NGAL and serum levels of CXCL16 for disease activity which when coupled with traditional disease markers, accurate prediction of underlying renal pathology and thus the clinical course of LN can be achieved. In addition, faster progression to CKD in patients with $\mathrm{LN}$ was best predicted by persistently high levels of uNGAL and sCXCL during follow-up.

\section{References}

1. Singh S, Wu T, Xie C, Vanarsa K, Han J, et al. (2012) Urine VCAM-1 as a marker of renal pathology activity index in lupus nephritis. Arthritis Research \& Therapy 14: R164.

2. Faurschou M, Starklint $H$, Halberg $P$, Jacobsen $S$ (2006) Prognostic factors in lupus nephritis: diagnostic and therapeutic delay increases the risk of terminal renal failure. J Rheumatol 33: 1563-1569.

3. Reyes-Thomas J, Blanco I, Putterman C (2011) Urinary Biomarkers in Lupus Nephritis. Clinical Reviews in Allergy \& Immunology 40: 138-150.

4. Brunner HI, Mueller M, Rutherford C, Passo MH, Witte D, et al. (2006) Urinary Neutrophil Gelatinase-Associated Lipocalin as a Biomarker of Nephritis in Childhood-Onset Systemic Lupus Erythematosus. Arthritis \& Rheumatism 54: 2577-2584.

5. Qin M, Guo $Y$, Jiang L, Wang X (2014) Elevated levels of serum CXCL16 in systemic lupus erythematosus; potential involvement in cutaneous and renal manifestations. Clin Rheumatol 33: 1595-1601.

6. Petri M, Orbai AM, Alarcón GS, Gordon C, Merrill JT, et al. (2012) Derivation and validation of the Systemic Lupus International Collaborating Clinics classification criteria for systemic lupus erythematosus. Arthritis and Rheumatism 64: 2677-2686.

7. Bertsias GK, loannidis JP, Aringer M, Bollen E, Bombardieri S, et al (2010) EULAR recommendations for the management of systemic lupus erythematosus with neuropsychiatric manifestations: report of a task of the EULAR standing committee for clinical affairs. Ann Rheum Dis 69: 2074-2823.

8. Weening JJ, D'agati VD, Schwartz MM, Seshan SV, Alpers CE, et al. (2004) The classification of glomerulonephritis in systemic lupus erythematosus revisited. Kidney International 65: 521-530.

9. Armitage P, Berry G, Matthews J (2002) Statistical methods in medical research. 4th edn. Oxford. Blackwell 4: 125.

10. Smith EM, Jorgensen AL, Midgley A, Oni L, Goilav B, et al. (2017) Internationa validation of a urinary biomarker panel for identification of active lupus nephritis in children. Pediatric Nephrology 32: 283-295.

11. Soni SS, Cruz D, Bobek I, Chionh CY, Nalesso F, et al. (2010) NGAL: a biomarker of acute kidney injury and other systemic conditions. International Urology and Nephrology 42: 141-150.

12. Hirsch R, Dent C, Pfriem H, Allen J, Beekman RH, et al. (2007) NGAL is an early predictive biomarker of contrast-induced nephropathy in children. Pediatric Nephrology 22: 2089.

13. Bolignano D, Coppolino G, Lacquaniti A, Nicocia G, Buemi M (2008) Pathological and Prognostic Value of Urinary Neutrophil Gelatinase-Associated
Lipocalin in Macroproteinuric Patients with Worsening Renal Function. Kidney Blood Press Res 31: 274-279.

14. Bolignano D, Coppolino G, Campo S, Aloisi C, Nicocia G, et al. (2007) Urinary neutrophil gelatinase-associated lipocalin (NGAL) is associated with severity of renal disease in proteinuric patients. Nephrology Dialysis Transplantation 23: 414-416.

15. Ding H, He Y, Li K, Yang J, Li X, et al. (2007) Urinary neutrophil gelatinaseassociated lipocalin (NGAL) is an early biomarker for renal tubulointerstitial injury in IgA nephropathy. Clinical Immunology 23: 227-234.

16. Yanaba K, Muroi E, Yoshizaki A, Hara T, Ogawa F, et al. (2009) Serum CXCL16 Concentrations Correlate with the Extent of Skin Sclerosis in Patients with Systemic Sclerosis. The Journal of Rheumatology 36: 1917-1923.

17. Bakr A (2005) Epidemiology treatment and outcome of childhood systemic lupus erythematosus in Egypt. Pediatric Nephrology 20: 1081-1086.

18. Salah S, Lotfy HM, Sabry SM, El Hamshary A, Taher H (2009) Systemic lupus erythematosus in Egyptian children. Rheumatology International 29: 1463.

19. Ali US, Dalvi RB, Merchant RH, Mehta KP, Chablani AT, et al. (1989) Systemic lupus erythematosus in Indian children. Indian Pediatrics 26: 868.

20. Uziel Y, Brik R, Padeh S (2007) Outcome of a national Israeli cohort of pediatric systemic lupus erythematosus. Lupus 16: 142-146.

21. Dung NT, Loan HT, Nielsen S, Zak M, Petersen FK (2012) Juvenile systemic lupus erythematosus onset patterns in Vietnamese children: a descriptive study of 45 children. Pediatric Rheumatology 10: 38.

22. Brunner HI, Gladman DD, Ibañez D, Urowitz MD, Silverman ED (2008) Difference in disease features between childhood-onset and adult-onset systemic lupus erythematosus. Arthritis and Rheumatism 58: 556

23. Bader-Meunier B, Armengaud JB, Haddad E, Salomon R, Deschênes G, et al. (2005) Initial presentation of childhood-onset systemic lupus erythematosus: A French multicenter study. The Journal of Pediatrics 146: 648-653.

24. Pluchinotta FR, Schiavo B, Vittadello F, Martini G, Perilongo G, et al. (2007) Distinctive clinical features of pediatric systemic lupus erythematosus in three different age classes. Lupus 16: 550-555.

25. Ramirez Gomez LA, Uribe Uribe O, Osio Uribe O, Grisales Romero H, Cardie $\mathrm{MH}$, et al. (2008) Childhood systemic lupus erythematosus in Latin America. The GLADEL experience in 230 children. Lupus 17: 596-604.

26. Suzuki M, Wiers KM, Klein-Gitelman MS, Haines KA, Olson J, et al. (2008) Neutrophil gelatinase-associated lipocalin as a biomarker of disease activity in pediatric lupus nephritis. Pediatric Nephrology 23: 403-412.

27. Bolignano D, Lacquaniti A, Coppolino G, Donato V, Campo S, et al. (2009) Neutrophil Gelatinase-Associated Lipocalin (NGAL) and Progression of Chronic Kidney Disease. Clinical Journal of the American Society of Nephrology: CJASN 4: 337

28. Susianti H, Iriane VM, Dharmanata S, Handono K, Widijanti A, et al. (2015) Analysis of urinary TGF- $\beta 1$, MCP-1, NGAL, and IL-17 as biomarkers for lupus nephritis. Pathophysiology 22: 65-71.

29. Torres-Salido MT, Cortés-Hernández J, Vidal X, Pedrosa A, Vilardell-Tarrés M et al. (2014) Neutrophil gelatinase-associated lipocalin as a biomarker for lupus nephritis. Nephrology Dialysis Transplantation 29: 1740-1749. 